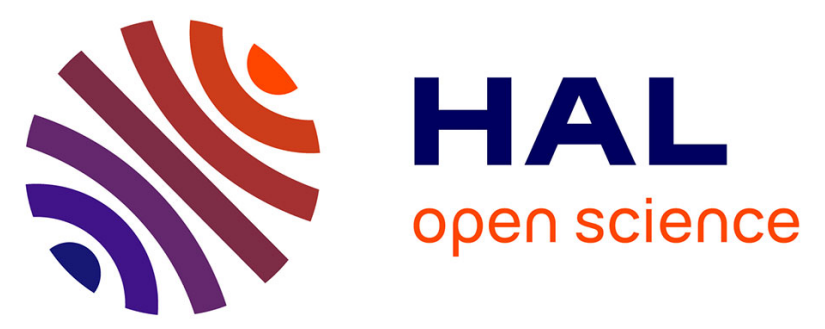

\title{
Comparison of Air Impaction and Electrostatic Dust Collector Sampling Methods to Assess Airborne Fungal Contamination in Public Buildings A BSTR ACT
} Anne-Cécile Normand, Stéphane Ranque, Carole Cassagne, Jean Gaudart, Kankoé Sallah, Denis-André Charpin, Renaud Piarroux

\section{To cite this version:}

Anne-Cécile Normand, Stéphane Ranque, Carole Cassagne, Jean Gaudart, Kankoé Sallah, et al.. Comparison of Air Impaction and Electrostatic Dust Collector Sampling Methods to Assess Airborne Fungal Contamination in Public Buildings A BSTR ACT. Annals of Occupational Hygiene, 2016, pp.161-175. 10.1093/annhyg/mev075 . hal-01307129

\section{HAL Id: hal-01307129}

https://hal-amu.archives-ouvertes.fr/hal-01307129

Submitted on 26 Apr 2016

HAL is a multi-disciplinary open access archive for the deposit and dissemination of scientific research documents, whether they are published or not. The documents may come from teaching and research institutions in France or abroad, or from public or private research centers.
L'archive ouverte pluridisciplinaire HAL, est destinée au dépôt et à la diffusion de documents scientifiques de niveau recherche, publiés ou non, émanant des établissements d'enseignement et de recherche français ou étrangers, des laboratoires publics ou privés.

\section{(ㅇ)(1) $\$$}

Distributed under a Creative Commons Attribution - NonCommercial - NoDerivatives 44.0 


\title{
Comparison of Air Impaction and Electrostatic Dust Collector Sampling Methods to Assess Airborne Fungal Contamination in Public Buildings
}

\section{Anne-Cécile Normand ${ }^{1 *}$, Stéphane Ranque ${ }^{1,2}$, Carole Cassagne ${ }^{1,2}$, Jean Gaudart ${ }^{3}$, Kankoé Sallah ${ }^{3}$, Denis-André Charpin ${ }^{4}$ and Renaud Piarroux ${ }^{1,2}$}

\author{
1.Parasitology and Mycology, Assistance Publique-Hôpitaux de Marseille, CHU Timone-Adultes, Marseille, France; \\ 2.Aix-Marseille University, UMR MD3 IP-TPT, Marseille, France; \\ 3.Aix-Marseille University, UMR912 (SESSTIM), INSERM/AMU/IRD, Marseille, France; \\ 4.Clinique des bronches, allergie et sommeil, Hôpital Nord, Inserm U1067 CNRS UMR1067, Marseille, France \\ *Author to whom correspondence should be addressed. Tel: +33 4913860 90; fax : +33 49138 49 58; e-mail: anne-cecile.normand@ap-hm.fr \\ Submitted 12 May 2015; revised 22 September 2015; revised version accepted 23 September 2015.
}

\begin{abstract}
Many ailments can be linked to exposure to indoor airborne fungus. However, obtaining a precise measurement of airborne fungal levels is complicated partly due to indoor air fluctuations and non-standardized techniques. Electrostatic dust collector (EDC) sampling devices have been used to measure a wide range of airborne analytes, including endotoxins, allergens, $\beta$-glucans, and microbial DNA in various indoor environments. In contrast, viable mold contamination has only been assessed in highly contaminated environments such as farms and archive buildings. This study aimed to assess the use of EDCs, compared with repeated air-impactor measurements, to assess airborne viable fungal flora in moderately contaminated indoor environments. Indoor airborne fungal flora was cultured from EDCs and daily air-impaction samples collected in an office building and a daycare center. The quantitative fungal measurements obtained using a single EDC significantly correlated with the cumulative measurement of nine daily air impactions. Both methods enabled the assessment of fungal exposure, although a few differences were observed between the detected fungal species and the relative quantity of each species. EDCs were also used over a 32-month period to monitor indoor airborne fungal flora in a hospital office building, which enabled us to assess the impact of outdoor events (e.g. ground excavations) on the fungal flora levels on the indoor environment. In conclusion, EDC-based measurements provided a relatively accurate profile of the viable airborne flora present during a sampling period. In particular, EDCs provided a more representative assessment of fungal levels compared with single air-impactor sampling. The EDC technique is also simpler than performing repetitive air-impaction measures over the course of several consecutive days. EDC is a versatile tool for collecting airborne samples and was efficient for measuring mold levels in indoor environments.
\end{abstract}

KEYWORDS: airborne fungal flora; electrostatic dust collector; filamentous fungi; indoor air; molds; public buildings 


\section{INTRODUCTION}

Individuals spend more than $80 \%$ of their lifetime indoors (Bernstein et al., 2008) exposed to a variety of airborne flora. Indoor exposure to molds has been associated with many medical conditions and a wide spectrum of clinical symptoms, including cough (Bush, 2008), various respiratory infections (Piecková and Wilkins, 2004; Haliki-Uztan et al., 2010; Hedayati et al., 2010; Kawel et al., 2011), skin conditions (Marcoux et al., 2009), irritations, toxic reactions (Fischer and Dott, 2003; McGinnis, 2004), headaches, and fatigue (Wålinder et al. 2005). In 2011, Mendell et al. have estimated that $\sim 10 \%$ of the world population experiences allergic reactions associated with mold exposure. Many fungal species, including those of the genera Aspergillus, Alternaria, Penicillium, and Cladosporium, are involved in allergic reactions (Dassonville et al., 2008; Bogomolova and Kirtsideli, 2009; Zuraimi et al., 2009; Cai et al., 2011; Hasnain et al., 2012). In a large scale study of primary school environments, Jacobs et al. (2014) have reported a link between dampness and respiratory symptoms, although the symptoms did not correlate with microbial levels upon targeted real-time PCR (RT-PCR) assessment of EDC samples. Exposure to indoor microbial flora have been shown to exert damaging effects on human health, such circumstances especially exacerbate asthma or wheezing (Mendell et al., 2011; Kanchongkittiphon et al., 2015). Inversely, exposure to Alpine farms indoor microbial flora have shown to be protective against asthma and allergies (Ege et al., 2011). Establishing direct relationships between fungal exposure and human health conditions is complicated (DeKoster and Thorne, 1995; Zuraimi et al., 2009), especially with a lack of standard sampling methods to measure indoor fungal levels (Méheust et al., 2013). Indoor fungal exposure levels at a given site vary greatly over time (Hyvärinen et al., 2001) depending on several parameters, such as humidity (Burge, 1985), season (Frankel et al., 2012a), and occupant activity during the few hours preceding sample collection. To overcome this lack of reproducibility, some authors have recommended collecting at least three air samples at different time points in each monitored room (Portnoy et al., 2004). Although increasing the number of samples circumvents the issue of reproducibility, this method is hardly practical for large-scale studies as the investigator must then remain in each of the studied rooms for at least $1 \mathrm{~h}$. To reduce the time required at each site, some studies have used an alternative approach in which dust settled on various surfaces, such as floors, chairs, and mattresses (Chao et al. 2002; Pitkäranta et al., 2008; Ege et al., 2011), was vacuum-collected, thereby enabling the analysis of microbial DNA or viable fungal diversity. However, these methods are not appropriate to assess dust that settles during a defined time period.

To overcome this latter issue, the Electrostatic dust collector (EDC) was used to collect settled dust for downstream analysis. EDCs consist of electrostatic cloths that function as the surface area for dust sedimentation. This particular dust collector allows for better control of sampling duration depending on the experiment. Results can be expressed in units per square meter per day and thus compared between studies.

The EDC method, which was first proposed by Noss et al. $(2008,2010)$ to analyze endotoxin and glucan levels, has also been used to study exposure to various allergens in indoor environments, such as farms, homes, schools, and veterinary units (Samadi et al., 2010; Zahradnik et al., 2011; Krop et al., 2014). This method has also been used to assess exposure to viable microorganisms in different types of buildings (e.g. dwellings and farms) (Normand et al., 2009; Frankel et al., 2012b). We have previously shown that EDC-based measurements in highly contaminated environments attenuate daily variations in airborne fungal contamination levels and subsequently enhance measurement reproducibility (Normand et al., 2009). Frankel et al. (2012b) have obtained similar results in a dwelling setting. Both studies showed a good correlation in fungi levels between EDC samples and airborne dust. Thus, Frankel et al. (2012b) have suggested that EDCs could be considered a surrogate for the air-impaction method to assess indoor microbial exposure.

To the best of our knowledge, an EDC-based assessment of exposure to viable fungi in public buildings has never been reported. In public environments, one may be chronically exposed to a moderate but consistent level of airborne fungi, as has been shown in a large number of schools by assessing certain fungal species via targeted RT-PCR and $\beta$-glucan levels (Jacobs et al., 2014).

The present study aimed to assess the use of EDCs to measure airborne fungal exposure in public 
structures and compare EDC and repeated air-impactor measurement capacity. We selected three types of buildings with two different kinds of human activity: (i) a small day care center, with no more than 15 children younger than 3 years old, (ii) a three-floor office building, and (iii) a small office building inside a hospital, where we intended to assess the influence of nearby ground excavations on the richness of indoor airborne fungal flora.

\section{MATERIALS AND METHODS}

\section{Preliminary experiment concerning the effects of freezing EDCs}

Prior to the main study, we performed an experiment in which we deposited nine EDCs in parallel in three different rooms of our Parasitology/Mycology laboratory in Marseille. After 14 days of exposure, collected dust was directly extracted from three EDCs per room as explained in the sample handling section. The remaining EDCs were frozen for 1 month $(3 \times 3$ EDCs) and 2 months $(3 \times 3$ EDCs $)$. They were then treated following the same protocol applied for the first nine EDCs.

\section{Preliminary experiment on reproducibility of EDC sampling}

To test the variability from one EDC to another, we deposited two EDCs in parallel in 13 rooms and treated them as described in the sample handling section.

\section{Main study's buildings locations}

The samples were collected in three different buildings located in the conurbation of Marseille (France): (i) a large office building in Aubagne, a city located 16 $\mathrm{km}$ east of Marseille; (ii) a five-room daycare center in Marseille, and (iii) a small office building at the University Hospital La Timone in Marseille. In both, the Aubagne office building and the Marseille daycare center, EDC measurements were compared with repeated air-impaction sampling. In the hospital office building, EDCs were used for long-term monitoring of mold exposure and in particularly to measure the effects of ground excavations and construction work carried out in the vicinity. The study did not involve the participation of the individuals occupying the study sites. The director of the offices and the respective city halls granted our team access to the facilities.
Eight rooms were randomly selected in the threestory office building in Aubagne as follows: three on the second floor, one on the first floor, three on the ground floor, and one in the basement. All five rooms of the one-story daycare center in Marseille were assessed.

In total, both EDC-based analysis and repeated airimpaction sampling were performed in 13 rooms at these two sites. Over the course of the two sampling campaigns, 176 air impaction and 16 EDC measurements were collected at the office building in Aubagne, and 105 air-impaction and 10 EDC measurements were collected at the daycare center in Marseille. The characteristics of the sampling campaigns performed at each building are detailed in Table 1 .

For the long-term EDC-based monitoring of airborne fungal contamination at the hospital office building, two offices were monitored: one opposite the construction site (Office 1) and the other facing the construction site (Office 2). The longitudinal study spanned a 32-month period, from May 2010 to February 2013, during which EDCs were replaced every 2 weeks in both offices, thereby yielding a total of 66 EDC measurements in each office.

\section{Environmental data}

Temperature and precipitation data were collected from the https://donneespubliques.meteofrance.fr/ website, using the Marignane forecast station (number 07650). The average temperature and total precipitation levels were calculated per sampling period for both the daycare center and the office building. The corresponding temperature and precipitation levels for the longitudinal study period are shown (Fig. 5a).

\section{Air-impaction sampling}

Air-impaction samplings were conducted every weekday (the office building and the daycare center were closed on Saturday and Sunday) at the same time each day (Table 1) using an MAS-100 NT Air-Sampler System (Merck). According to the standard NF EN ISO 14698 protocol, 3301 ( $3 \mathrm{~min}$ and $18 \mathrm{~s}$ at a 100 $1 \mathrm{~min}^{-1}$ flow) of air were impacted on a Sabouraud Chloramphenicol Gentamicin (OXOID) agar plate, which was incubated for 7 days at $30^{\circ} \mathrm{C}$.

\section{EDC sampling}

Each EDC had a standardized surface size of $10 \times 20 \mathrm{~cm}$ $\left(0.02 \mathrm{~m}^{2}\right)$. EDCs were heat sterilized and deposited for 


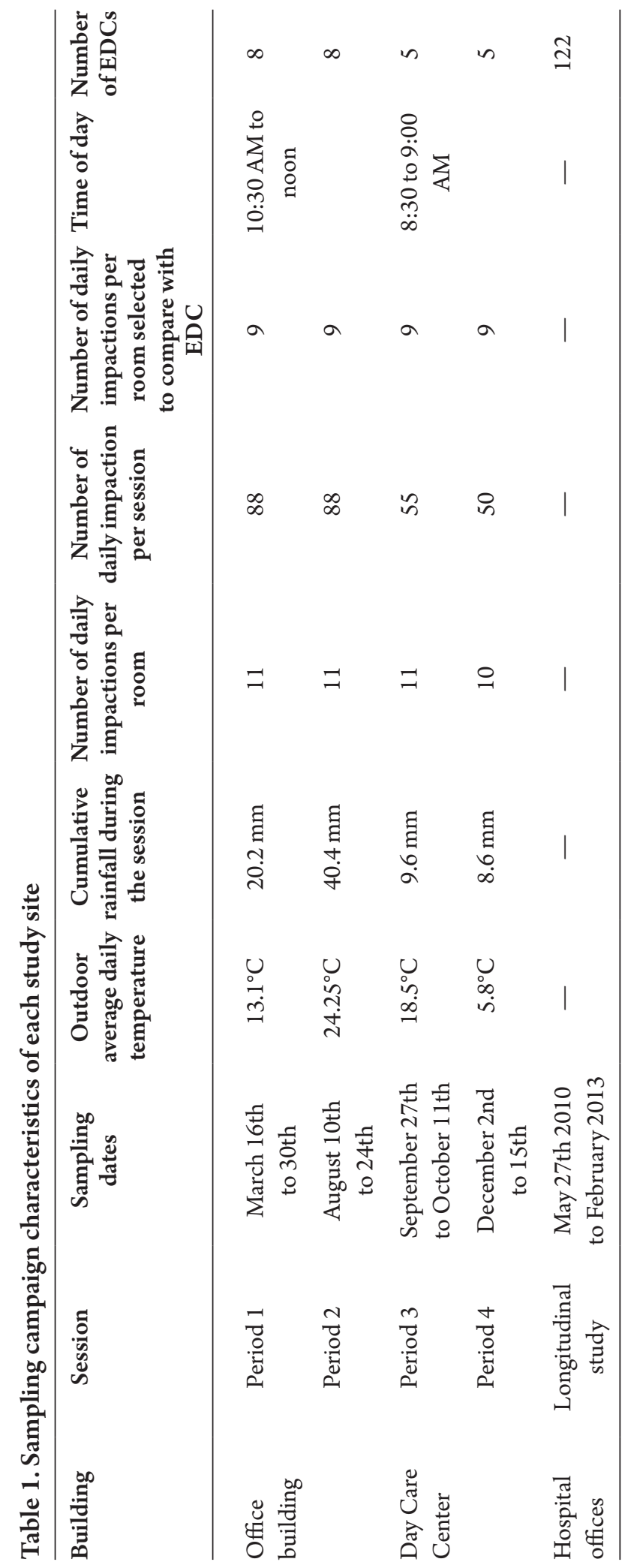


14-15 days on a cleaned plate that was located at varying heights (between 1.30 and $1.60 \mathrm{~m}$ ), with the exception of one room in which no support below $2 \mathrm{~m}$ was available. The EDCs were placed far from windows and sinks to avoid both air drafts and humidity. The inhabitants of the various offices were informed not to change their habits, especially regarding opening windows in the studied rooms. To compare the two methods, the air impactor was positioned each day on the same surface and in close proximity to the EDC.

\section{Sample handling}

As we expected low fungal contamination levels of the surveyed rooms, a concentration step was included to adapt the microbial culture protocol described by Normand et al. (2009) for farm exposure assessment. Briefly, after each 14-day exposure period, the EDCs were collected in a sterile plastic bag and stored at $-20^{\circ} \mathrm{C}$ until they were analyzed (as soon as possible, with a 1-day to 1-month delay). The EDCs were washed with $30 \mathrm{ml}$ of Tween $80(0.1 \%)$ in a blender bag (Gosselin, France) and then washed vigorously in a stomacher (Homogenius HG400, Mayo international, Italy) for $10 \mathrm{~min}$. The resulting solution was transferred into two $15-\mathrm{ml}$ conical tubes (BD Falcon $\left.^{\mathrm{Tx}}\right)$. The two conical tubes were centrifuged for $10 \mathrm{~min}$ at $3500 \mathrm{rpm}$, the supernatant was then discarded, and the pellets were resuspended in $1 \mathrm{ml}$ Tween 80 (0.1\%). The two resuspended pellets, corresponding to one EDC sample, were pooled, and $200 \mu$ l of the pooled solution was then inoculated onto a Sabouraud Chloramphenicol Gentamicin agar plate (OXOID, Dardilly, France). The plates were incubated for seven days at $30^{\circ} \mathrm{C}$. The fungal colonies were identified based on their macroscopic and microscopic characteristics (De Hoog, 2001). Colony forming units (CFUs) were quantified for each fungal taxon.

\section{Statistical analysis}

Throughout the experiment, comparisons between sampling devices (EDC versus air-impaction) were performed by assessing the number of fungal taxa/

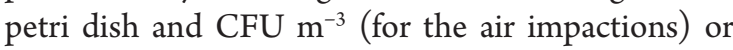
CFU m ${ }^{-2}$ day $^{-1}$ (for the EDCs) to characterize the environments. Data comparisons regarding the preliminary experiments were performed using the intraclass correlation coefficient (ICC) for both CFU counts and the number of fungal taxa. Statistical dispersion of the data for each method is represented using boxplot graphs, which depict the median as well as the first and third quartiles of each data set.

We defined the sum of air-impaction measurements carried out in a given location during a sampling session, which most accurately reflects the true levels of fungal contamination, as the reference standard value to assess the accuracy of each measurement performed during the study. However, to avoid comparing data that are included in the reference standard with the reference standard, we removed the first air-impaction measurement from the cumulative values to assess both air impaction and EDC versus the reference standard. In order to standardize the number of air impactions we analyzed only nine measures in periods 1,2 , and 3 . As each method has distinct units, the correlation between the CFUs recovered using EDCs and those recovered via air-impaction was assessed using Spearman's rank correlation coefficient. Both CFU counts and number of fungal taxa were compared between the first single air-impaction (performed on the first day of the study), the sum of repeated air-impaction samples and EDC samples. The correlation between fungal $\mathrm{CFU}$ counts and fungal taxa richness measured with EDC or repeated air-impaction was estimated using intra-class correlation coefficients (ICC).

The accuracy of the EDC and air-impaction methods was determined based on the capacity to detect significant differences in distinct locations (i.e. daycare center versus office building) and during distinct periods (i.e. summer versus winter). Spatial and temporal effects were tested using the Kruskal-Wallis test.

The Wilcoxon test was used to compare the fungal contamination levels at the two offices located at the hospital of Marseille. Three different construction periods were considered: excavation of the foundation (LP1), exterior wall construction (LP2), and interior construction (LP3). The Kruskal-Wallis test was used to analyze the effect of the type of work performed in the new building on fungal CFU counts, adjusted on the number of analyzed samples. Statistical analyses were performed using R software (version 3.1.0; www.rproject.org); all tests were two-tailed, with $\alpha<0.05$.

\section{RESULTS}

\section{Preliminary experiment concerning the effects of freezing EDCs}

The experiment showed a reproducibility of both fungal taxa richness and CFU counts, with ICC $=0.61$ [0.22-0.85] and $0.9495 \% \mathrm{CI}[0.33,0.99]$, respectively. 
In this preliminary experiment, we found that some of the species recovered, such as Aspergillus fumigatus, Aspergillus nidulans, Aspergillus niger, and the Penicillium spp. taxa, were more likely than others to resist the freezing process (data not shown).

\section{Preliminary experiment on reproducibility of EDC} sampling

The reproducibility of EDC sampling is good, with an ICC of $0.68[0.36,0.89](P=0.0058)$ and 0.97 [0.91, $0.99](P=0.0004)$ for fungal taxa richness and CFU counts, respectively.

\section{Species identification}

The 34 fungal species or taxa identified were grouped into 18 taxa (Table 2).

Characteristics of the fungal contamination of the two buildings using the three different methods are shown in Table 3.

\section{Day-to-day variability in air-impaction sampling} As illustrated in Fig. 1, air-impaction sampling showed dramatic day-to-day variations in total airborne fungal flora levels.

The cumulative fungal airborne contamination measurements calculated by aggregating nine iterative air-impaction samples as well as via the EDC collection method are provided in Fig. 2. Cumulative airborne fungal contamination measurements highlighted obvious differences in fungal airborne levels depending on the building location and study period (Fig. 2). Although the taxa recovered by both sampling methods were comparable, we found differences in the fungal composition of the samples when comparing cumulative air samples with EDC samples.

\section{Number of fungal taxa cultured from EDC or air-impaction samples}

EDC and air-impaction fungal exposure measurements displayed few differences between detected fungal species. Most taxa were cultured from both EDC and air-impaction samples, albeit in varying relative quantities. Only the Scedosporium and Scopulariopsis taxa were isolated once (one CFU each) from airimpaction samples and never from the EDC samples. The most abundant fungal taxa recovered using both methods were Cladosporium and Penicillium. However, Cladosporium molds were represented to a
Table 2. Species included in the fungal taxa recovered in this study

\begin{tabular}{|c|c|}
\hline Taxa & Species \\
\hline Alternaria & Alternaria spp. \\
\hline Aspergillus flavi & Aspergillus flavus \\
\hline Aspergillus fumigati & Aspergillus fumigates \\
\hline Aspergillus nidulantes & Aspergillus nidulans \\
\hline Aspergillus nigri & Aspergillus niger \\
\hline Aspergillus terrei & Aspergillus terreus \\
\hline Other Aspergillus & $\begin{array}{l}\text { A. clavatus, A. hollandicus, A. ustus, } \\
\text { A. versicolor, Aspergillus spp. }\end{array}$ \\
\hline Chrysonilia & Chrysonilia spp. \\
\hline Cladosporium & $\begin{array}{c}\text { Cladosporium spp., } \\
\text { Cladophialophora spp., Phialophora } \\
\text { spp. }\end{array}$ \\
\hline Other Fungi & $\begin{array}{c}\text { Unidentified fungi, } \\
\text { Hyalohyphomyces spp., } \\
\text { Phaeohyphomyces, Ulocladium } \\
\text { spp., Yeast }\end{array}$ \\
\hline Fusarium & Acremonium spp., Fusarium spp. \\
\hline Mucorales & $\begin{array}{l}\text { Botrytis cinerea, Mucor spp., } \\
\text { Rhizopus spp. }\end{array}$ \\
\hline Penicillium & Penicillium spp., Paecilomyces spp. \\
\hline Scedosporium & Scedosporium spp. \\
\hline Scopulariopsis & Scopulariopsis spp. \\
\hline Scytalidium & Scytalidium spp. \\
\hline Sordariales & $\begin{array}{l}\text { Chaetomium spp., Phoma spp., } \\
\text { Microascus spp. }\end{array}$ \\
\hline Trichoderma & Trichoderma spp. \\
\hline
\end{tabular}

greater extent in the EDC samples compared with the cumulative air-impaction samples, specifically for the daycare center in Marseille. Thus, the number of positive rooms corresponding to each of the studied fungal taxa (based on presence/absence) measured via EDC or cumulative air-impaction was highly correlated $(r=0.90)$. ICC between EDC and cumulative airimpaction measures were $0.45[0.19,0.74]$ and 0.15 $[0.01,0.78]$ for fungal taxa richness and CFU counts, respectively. 
Table 3. Description of the fungal contamination as assessed by using the three methods: single air impaction, cumulative air impactions, and EDC

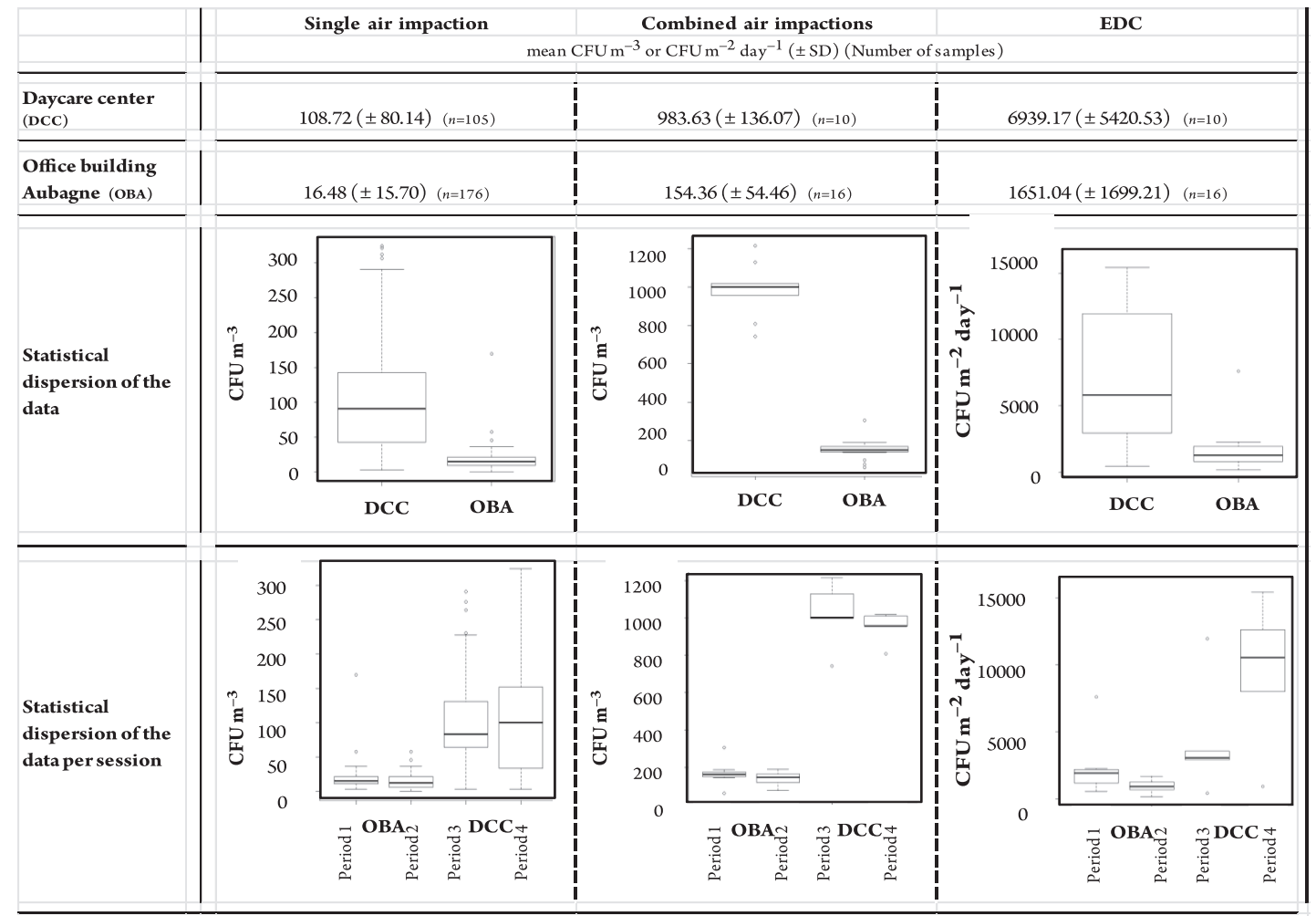

For statistical dispersion, the bottom and top of the boxplots are the lower and upper quartiles, respectively; the dark band represents the median; and the ends of the whiskers represent the lowest datum included in the 1.5 interquartile range (IQR) of the lower quartile and the highest datum included in the $1.5 \mathrm{IQR}$ of the upper quartile.

As shown in Fig. 3, it was necessary to aggregate the results of at least three consecutive days of airimpaction measurements to detect a number of fungal taxa comparable to that obtained with one EDC measurement. The figure also shows that the number of fungal taxa obtained after nine cumulative airimpaction measurements is almost 2-fold higher than that obtained with one EDC measurement.

\section{Measure of the number of fungal taxa and CFU counts}

For both number of fungal taxa and CFU counts, the correlation between EDC and air-impaction measurements increased with the number of cumulative airimpaction measurements (Fig. 4).

Due to the poor reproducibility of individual air sampling measures, EDC-based measurements were poorly correlated with single sampling results (number of fungal taxa: $r=0.19$ and CFU count: $r=0.4$ ), whereas a higher correlation was obtained when comparing a single EDC measurement with the cumulative air-impaction measurements (number of fungal taxa: $r=0.50$ and CFU counts: $r=0.74$ ).

\section{Comparing EDC to the standard}

Comparing EDC measurements with the reference standard yielded a significant Spearman correlation coefficient of $0.63(P=0.0001)$. Regarding the number of fungal taxa, the Spearman correlation coefficient was only significant between EDC measurements and the reference standard with a Spearman correlation coefficient of $0.45(P=0.0139)$.

\section{Spatial and temporal analyses of each sampling method}

The comparison between different buildings or seasons showed that the three approaches (first airimpaction, cumulative air-impactions, and EDC) 

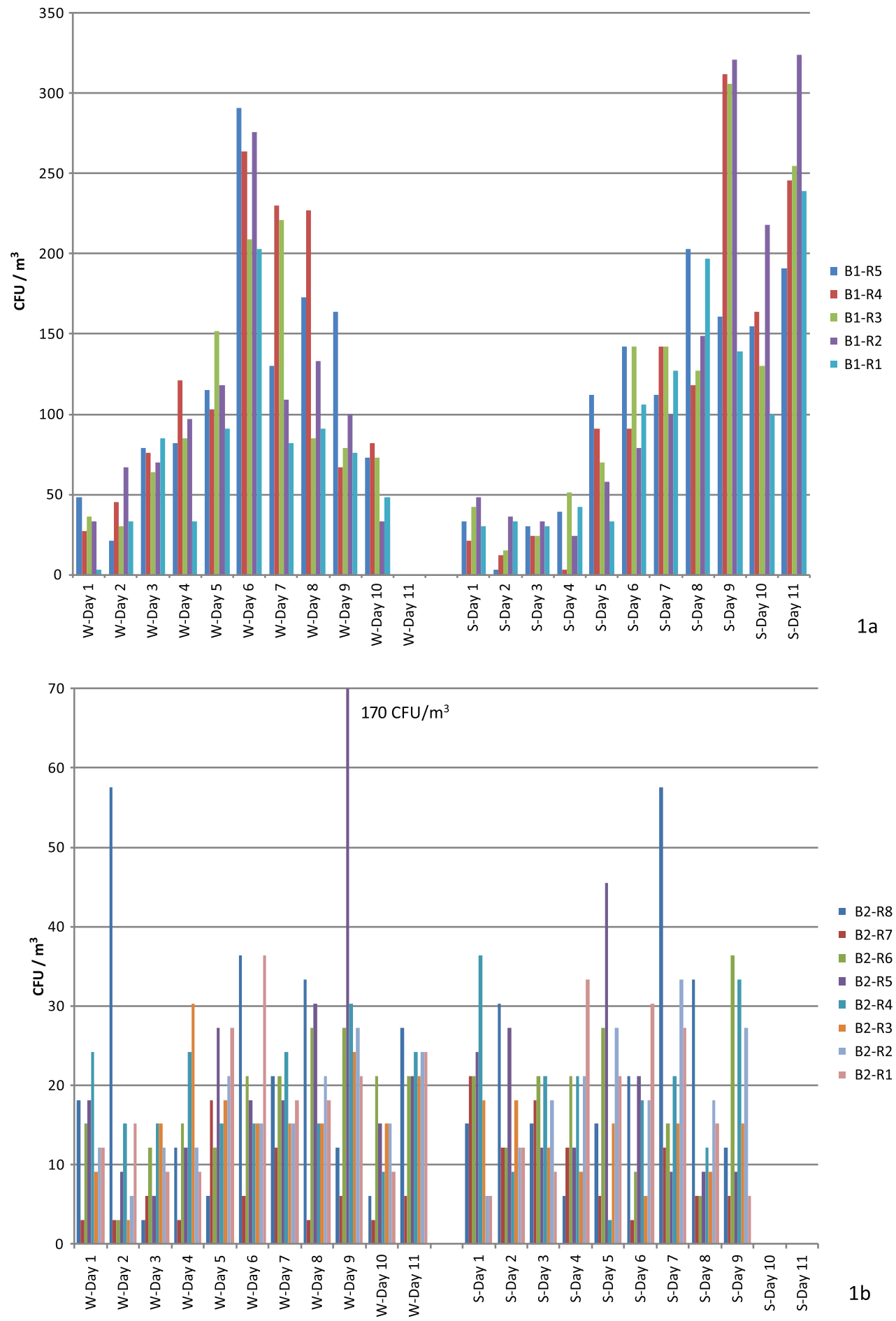

Figure 1 Daily variations in fungal contamination levels in the 13 different rooms. (a) B1 corresponds to the daycare center in Marseille (scale from 0 to $350 \mathrm{CFU} \mathrm{m}{ }^{-3}$ ); (b) B2 corresponds to the office building in Aubagne (scale from 0 to $70 \mathrm{CFU} \mathrm{m}^{-3}$ with an outlier at $\left.170 \mathrm{CFU} \mathrm{m}^{-3}\right)$. Total fungal contamination $\left(\mathrm{CFU} \mathrm{m}{ }^{-3}\right)$ is reported for each sampling day for the 13 rooms. S-Day 1 to S-Day 11 corresponds to the summer samplings; W-Day 1 to W-Day 11 corresponds to the winter samplings. 

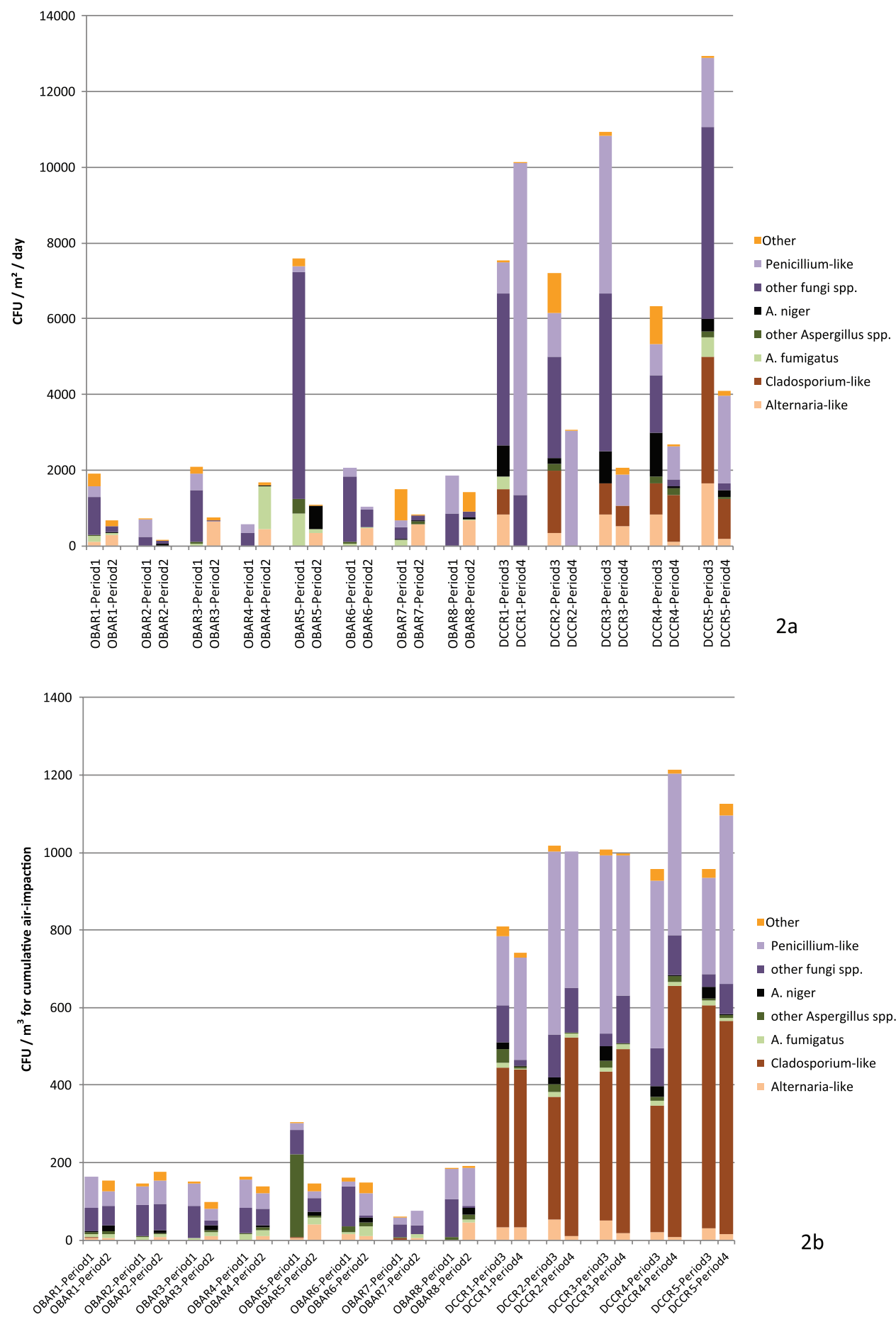

Figure 2 Fungal distribution measured with EDCs and cumulative air-impaction measurements. DCC corresponds to the daycare center in Marseille; OBA corresponds to the office building in Aubagne; (a) The results of the fungal taxa identified using EDCs during the different periods for the 13 rooms; (b) The results of the fungal taxa identified using cumulative air-impaction measurements during the different periods for the 13 rooms. 


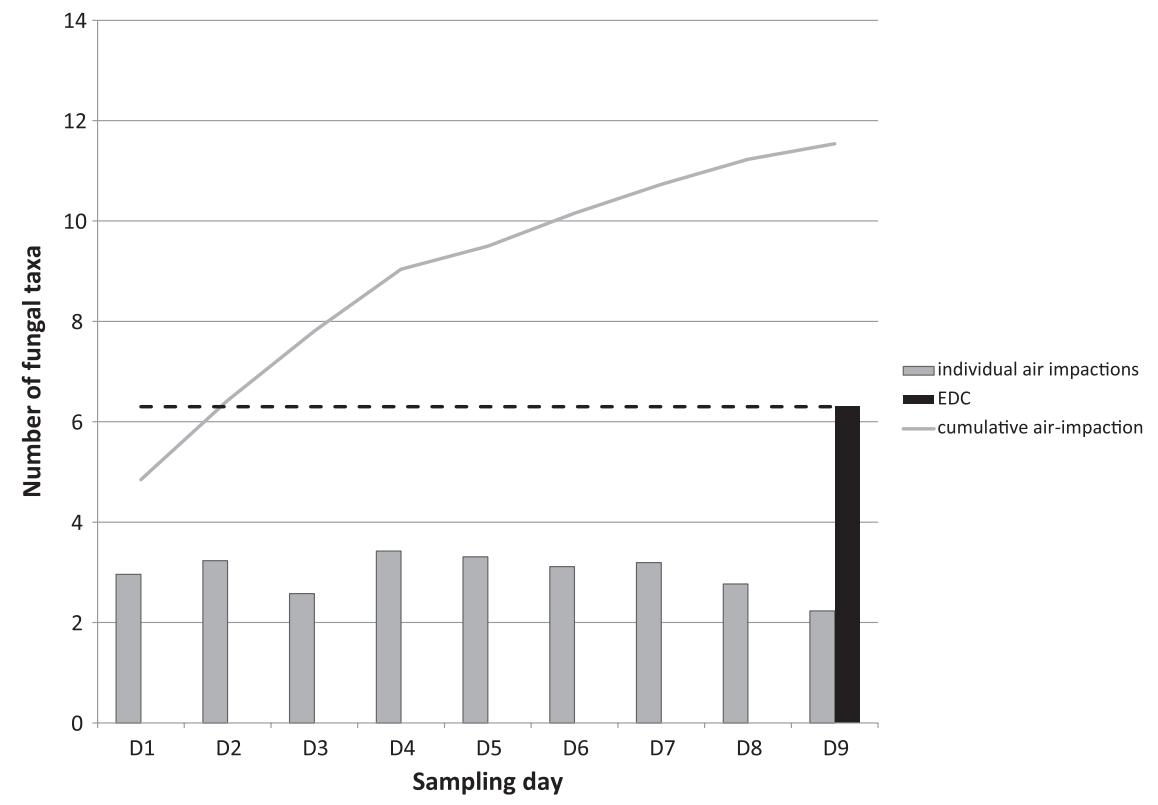

Figure 3 Comparison of the number of fungal taxa measured using EDC or cumulative air-impaction samples. The variation in the number of fungal taxa identified using the two methods is shown. The horizontal dotted line corresponds to the value obtained after 14 days of exposure using the EDC.

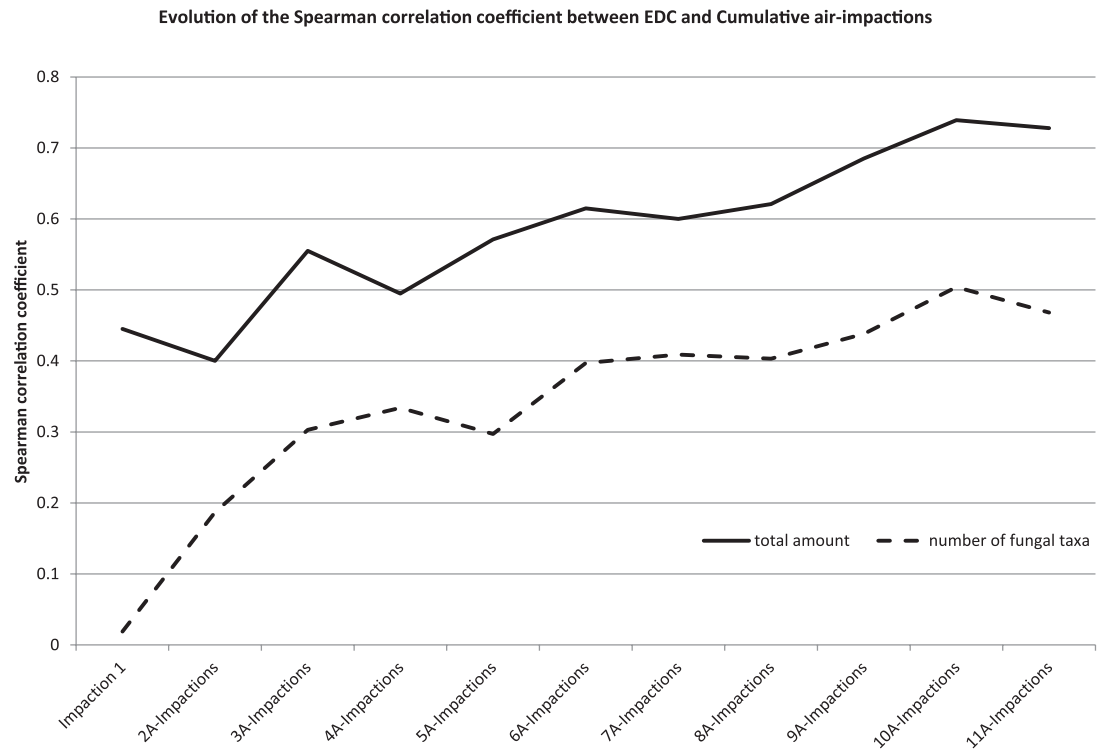

Figure 4 Effect of the number of cumulative air-impaction samples on the Spearman correlation coefficient between measurements using EDC or air-impaction. A-Impactions: cumulative air-impaction results. The dotted line corresponds to the number of fungal taxa identified, and the solid line corresponds to the total fungal CFU counts.

highlighted spatial differences in both fungal counts and number of fungal taxa (with the exception of the number of fungal taxa obtained with the first air-impaction). However, regardless of the method, we did not observe a significant seasonal pattern, with the exception of one parameter (number of fungal taxa measured via cumulative air-impactions) (Table 4). 
Table 4. Comparison of spatial and temporal effects on the fungal taxa richness or fungal counts measured with the three sampling methods (EDC, cumulative air impactions, and first air impaction)

\begin{tabular}{lcccc}
\hline Comparison & & EDC & Cumulative AI & First AI \\
\hline First versus second sampling & Fungal count & 0.54 & 0.46 & 0.29 \\
session & Number of fungal taxa & 0.17 & 0.0025 & 0.26 \\
$\begin{array}{l}\text { Daycare center versus office } \\
\text { building }\end{array}$ & Fungal count & 0.005 & 0.00003 & 0.003 \\
\hline
\end{tabular}

The statistically significant Kruskal-Wallis $P$ values are highlighted in gray ( $\mathrm{AI}=$ air impaction).

\section{Long-term monitoring of indoor airborne contaminants using EDCs}

Airborne fungal contamination levels in the two offices in the hospital building did not significantly differ when considering the entire 32-month period $(P=0.25)$ or when considering the first (LP1) and the second periods (LP2) individually ( $P=0.58$ and $P=0.87$, respectively). However, the contamination levels of Office 1 were significantly lower than those of Office 2 during the third period (LP3) $(P<0.005)$. Airborne contamination levels were significantly higher during LP1 compared with the two other periods for both Office $1\left(P<10^{-7}\right)$ and Office $2\left(P<10^{-5}\right)$.

Long-term monitoring of indoor-air fungal contamination at the two hospital offices highlighted a peak in fungal levels during the excavation of the foundation located less than $100 \mathrm{~m}$ from the two monitored offices. The peak in airborne fungal levels associated with this construction site was markedly higher for the office facing the construction site than the office oriented in the opposite direction. Interestingly, this increase in airborne fungal contamination was caused by a peak in Aspergillus sp. levels in the office facing the building site and two peaks in Cladosporium sp. levels. The first peak in Cladosporium sp. levels occurred in both offices, and the second peak occurred only in Office 2, which faced the construction site (Fig. 5). With the exception of a unique Aspergillus sp. peak during the autumn of 2012, followed by a wider but lower peak of Penicillium sp. in both offices, airborne fungal contamination levels steadily decreased after the excavation work was completed.

\section{DISCUSSION}

In the present study, we compared indoor airborne fungal contamination levels in public buildings using two different methods, one based on passive settlement and other based on active impaction. We showed that EDC is a method that enables a valuable and effective assessment of the fungal contamination profile in a public building, with results comparable to three consecutive days of airimpaction measurements. By comparing the first single air-impaction measurement with both EDC and cumulative air-impaction results, we confirmed the findings of previous studies highlighting the poor reliability of a single air-impaction measurement (Tovey and Green, 2005; Normand et al., 2009; Reboux et al., 2009). However, as we observed a significant correlation in fungal levels using EDC sampling and repeated air-impaction, these methods provide more comparable results than that obtained via a single air-impaction measurement. Furthermore, similarities between the fungal species isolated from EDCs and multiple air-impaction samples suggest that EDCs yield a fairly representative profile of the airborne fungal flora present during the sampling period.

Regardless of the method used, we identified a fungal signature for each investigated building. Indeed, the EDC and cumulative air-impaction sampling results highlighted the differences between the fungal airborne levels in the two public buildings in Aubagne and Marseille, which demonstrated higher fungi exposure levels at the daycare center compared with the office building. A similar trend was observed with the first air-impaction measurements. Regarding the EDC results, the concentration levels of fungal spores found in our study (range $=1651-6939 \mathrm{CFU} \mathrm{m}^{-2}$ day $^{-1}$ ) were comparable to those reported in a study of 27 Copenhagen residences (range $=206-6515$ CFU m $^{-2}$ day $^{-1}$ ) (Madsen et al., 2012); therefore, fungal loads determined using EDCs can be compared between studies. In a previous study carried out in Alpine farm buildings known to be highly contaminated with molds, EDC measurements were accurate enough to 


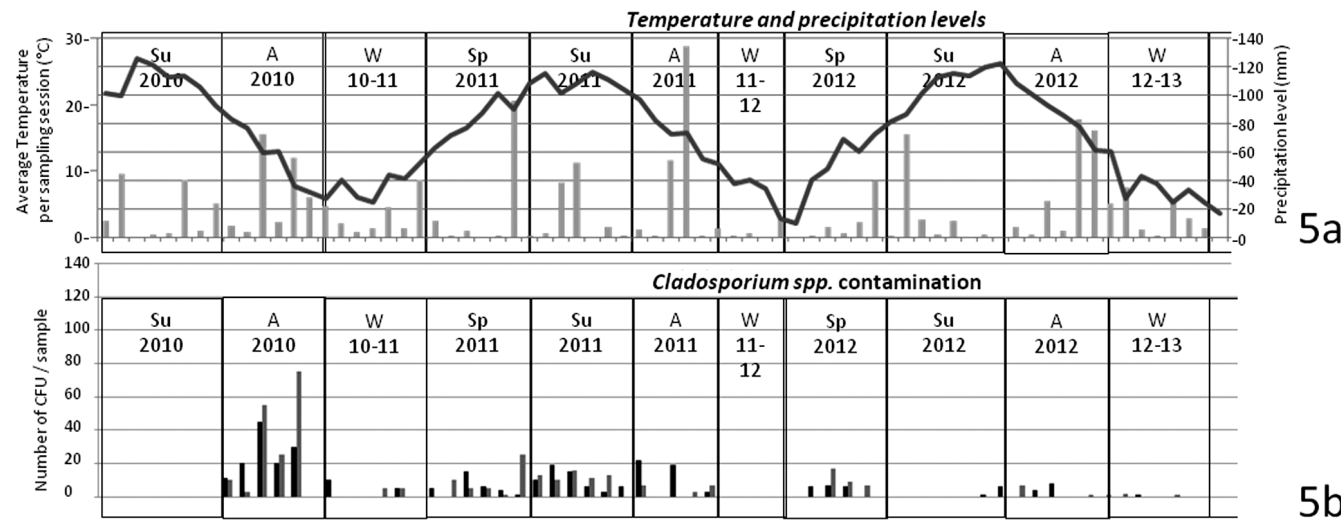

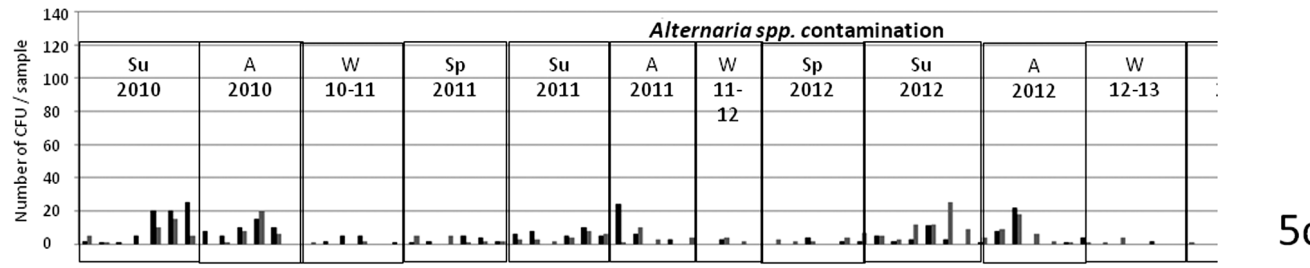

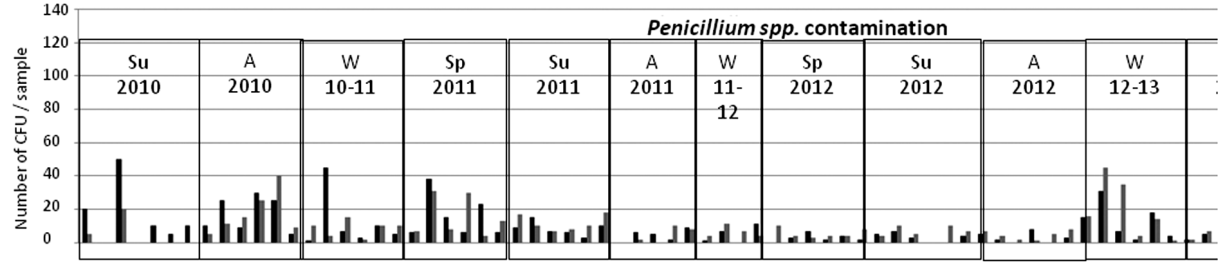

$5 c$

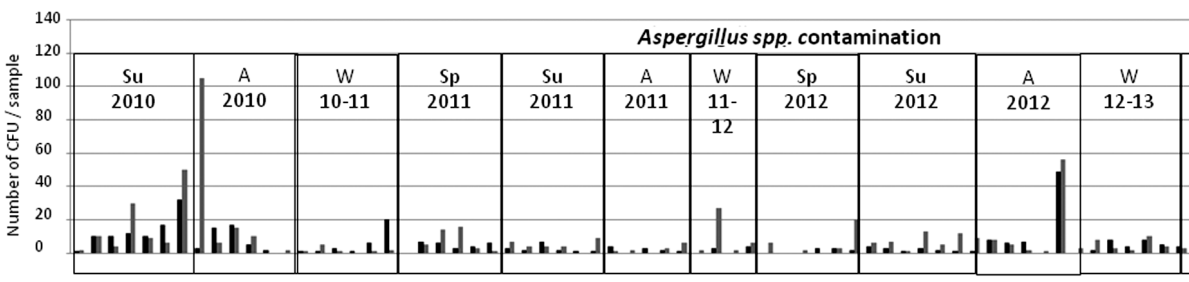

140 Total fungal contamination

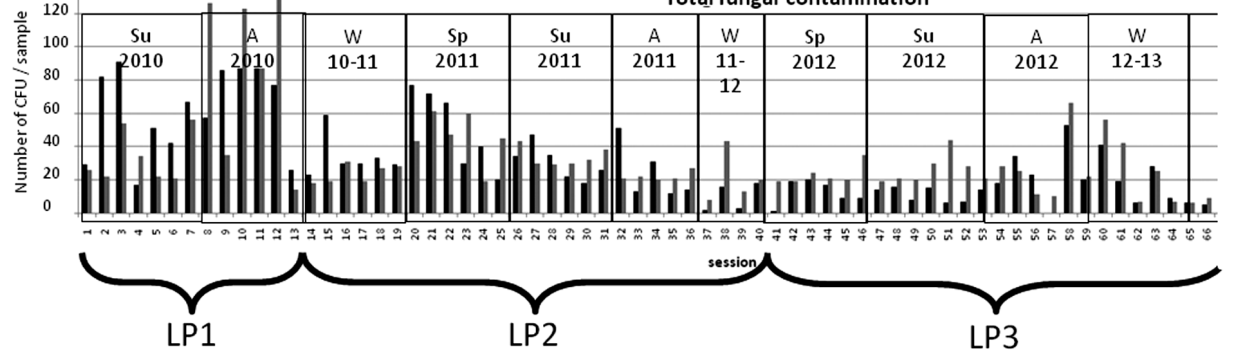

$5 e$

Figure 5 Fungal contamination patterns in an office building over the course of 3 years. LP1: Foundation excavation of a new building facing office 2; LP2: construction of the exterior wall of the new building; LP3: interior construction of the new building. (a) Temperature (line) and precipitation (bars) levels (average of 2 weeks) during the study period. (b-e) The fungal counts of four different taxa (respectively Cladosporium spp., Alternaria spp., Penicillium spp. and Aspergillus spp.). (f) Corresponds to the total cultivable fungi count in the two offices. 
highlight significant correlations between the microbial counts in the dwellings and the corresponding animal sheds and barns (Normand et al., 2011). EDCs have also been used to detect a reduced risk of asthma associated with exposure to specific mold taxa (Ege et al., 2011). The longitudinal arm of our study showed that using several EDCs in different rooms of a building could highlight variations between the exposure levels in the different rooms. Such an approach may be of great interest when investigating the contamination source for patients presenting with clinical conditions possibly associated with fungal exposure. Furthermore, EDCs are also suited to measure indoor microbial contamination for extended periods (i.e. months or years), as they can easily be used to monitor 2-week periods with minimal hands-on sampling time.

EDC technology is also compatible with a variety of downstream mold quantification methods. Indeed, EDCs have been applied to evaluate the microbial load of dwellings in a large survey (Rocchi et al., 2015a). This method has also been coupled with Aspergillus fumigatus qPCR to assess the Aspergillus fumigatus load in the homes of cystic fibrosis patients using microorganism DNA (Rocchi et al., 2015b) extracted from indoor air samples. Using EDCs, qPCR, and culture methods Roussel et al. (2012) have found a link between health symptoms and fungal load in an archive building. The study showed that the major factor associated with symptoms in this particular environment was not airborne fungus, but rather contact with moldy books. Madsen et al. (2012) have shown that several factors should be considered when using EDCs to assess microorganism contamination levels in a home, including the presence of occupants, the season, and the position of the device. As there was a frequent turnover of inhabitants in both buildings, we could not assess the presence of occupants; however, we carefully monitored the test season and the position of the sampling devices.

Many authors tend to agree that assessing the indoor fungal contamination of a building is technically complicated (DeKoster and Thorne, 1995; Hyvärinen et al., 2001; Zuraimi et al., 2009; Frankel et al., 2012a; Méheust et al., 2013; Jacobs et al., 2014). Various studies have assessed several environmental markers, but few studies focused on cultivable fungal species. Currently, no range of microorganism concentrations has been established either to classify insalubrious buildings or to link clinical conditions and/or symptoms with microbial airborne load, regardless of the investigated building and sampling method. In general, EDCs can be used in indoor environments, but may not be suitable to measure sporadic exposure of high fungal loads, such as those that occur in agricultural work environments. An air-impactor is instead recommended in this type of setting. Furthermore, EDCs are not suited to measure the exposure of a given individual to variable levels of fungus due to frequent changes in activity throughout the day. In this setting, a portable device is more appropriate.

We aimed to overcome some limitations of our study by performing key preliminary experiments. We subsequently deduced that there is good reproducibility for the CFU counts, albeit with a non-negligible loss of diversity, between duplicate EDC measurements. Thus, the number of fungal taxa, which varies between two EDC replicates, is less likely to be comparable with those recovered using other sampling methods, such as air-impactions or portable devices. A longer exposure time of the EDC might allow an homogenization of the settling species as proposed in other studies (Jacobs et al., 2014; Krop et al., 2014; Rocchi et al., 2015b). The second preliminary study showed that freezing EDCs had limited effect on cultivable fungi. However, we cannot exclude that freezing resulted in a reduction in fungal taxa richness, which may explain the differences in the ranges of species between EDC and air impactions (shown in Fig. 3), as proposed by Madsen et al. (2012). We did not observe a pattern in fungal viability before and after freezing, as some that taxa were identified after the freezing process were not found before and vice-versa, although Aspergillus fumigatus, Aspergillus nidulans, Aspergillus niger, and the Penicillium spp taxa viability did not seem to be hampered by the freezing process. Moreover, a similar freezing step has been applied in several studies to avoid the simultaneous cultivation of a large number of samples (Ege et al., 2011; Normand et al., 2011).

In this study, we did not perform an exhaustive characterization of the fungal environment of the various buildings. We rather aimed to compare the different methods using one culture media instead of several culture media to ensure the recovery of a diverse spectrum of viable fungi. We are aware that our reading of the fungal taxa richness measured using EDC samples may be altered when a high 
fungal load is obtained. One way to prevent this issue in further analyses would be to systematically perform one or two additional dilutions of the EDC extraction liquid. In conclusion, EDCs are a versatile tool that enables the collection and analysis of various environmental parameters (e.g. microbial DNA, cultivable microorganisms, and various analytes) and appears efficient for measuring mold contamination levels in indoor environments. This study shows that EDCs can be used in environments that are moderately contaminated with fungal spores, in which individuals spend significant time and a high level of human activity occurs. In such environments, EDCs can be used in addition to other available sampling methods, thereby allowing for a fairly reliable estimation of airborne mold exposure levels. In this study, we showed that a single EDC measurement is comparable to the sum of several air-impaction measurements. Therefore, EDCs provide a more precise estimation of the fungal exposition of individuals in public buildings compared with a single air impaction. The relatively short hands-on time required for EDC sampling is particularly advantageous in the setting of large cohort surveys, which would otherwise require numerous investigators to achieve a comparably precise measure of the airborne fungal levels using air impaction. The development of these EDC devices may be useful in providing new insights into airborne fungal exposure-linked diseases and helping to establish a threshold to assess relationships between ailment and environment.

\section{FUNDING}

Conseil Régional de PACA and the Agence Régionale de Santé in the context of a network of indoor air quality experts (EQAIR).

\section{ACKNOWLEDGEMENTS}

We especially thank Sandy Moore for proofreading the English version of the manuscript.

\section{DECLARATION}

The authors declare no conflict of interest relating to the material presented in this article. Its contents, including any opinions and/or conclusions expressed, are solely those of the authors.

\section{REFERENCES}

Bernstein JA, Alexis N, Bacchus $\mathrm{H}$ et al. (2008) The health effects of non-industrial indoor air pollution. J Allergy Clin Immunol; 121: 585-91.

Bogomolova E, Kirtsideli I. (2009) Airborne fungi in four stations of the St. Petersburg Underground railway system. Int Biodeter Biodegr; 63: 156-60.

Burge HA. (1985) Fungus allergens. Clin Rev Allergy; 3: 319-29.

Bush RK. (2008) Indoor allergens, environmental avoidance, and allergic respiratory disease. Allergy Asthma Proc; 29: 575-9.

Cai G-H, Mälarstig B, Kumlin A et al. (2011) Fungal DNA and pet allergen levels in Swedish day care centers and associations with building characteristics. J Environ Monit; 13: 2018-24.

Chao HJ, Milton DK, Schwartz J et al. (2002) Dustborne fungi in large office buildings. Mycopathologia; 154: 93-106.

Dassonville C, Demattei C, Detaint B et al. (2008) Assessment and predictors determination of indoor airborne fungal concentrations in Paris newborn babies' homes. Environ Res; 108: 80-5.

DeKoster JA, Thorne PS. (1995) Bioaerosol concentrations in noncomplaint, complaint, and intervention homes in the Midwest. Am Ind Hyg Assoc J; 56: 573-80.

Ege MJ, Mayer M, Normand AC et al. (2011) Exposure to environmental microorganisms and childhood asthma. $N$ Engl J Med; 364: 701-9.

Fischer G, Dott W. (2003) Relevance of airborne fungi and their secondary metabolites for environmental, occupational and indoor hygiene. Arch Microbiol; 179: 75-82.

Frankel M, Bekö G, Timm M et al. (2012a) Seasonal variations of indoor microbial exposures and their relation to temperature, relative humidity, and air exchange rate. Appl Environ Microbiol; 78: 8289-97.

Frankel M, Timm M, Hansen EW et al. (2012b) Comparison of sampling methods for the assessment of indoor microbial exposure. Indoor Air; 22: 405-14.

Haliki-Uztan A, Ateş M, Abaci Ö et al. (2010) Determination of potential allergenic fungal flora and its clinical reflection in suburban elementary schools in Izmir. Environ Monit Assess; 168: 691-702.

Hasnain SM, Akhter T, Waqar MA. (2012) Airborne and allergenic fungal spores of the Karachi environment and their correlation with meteorological factors. J Environ Monit; 14: 1006-13.

Hedayati MT, Mayahi S, Denning DW. (2010) A study on Aspergillus species in houses of asthmatic patients from Sari City, Iran and a brief review of the health effects of exposure to indoor Aspergillus. Environ Monit Assess; 168: 481-7.

Hoog GS de, Cuarro GJ, Figueras MJ (eds). (2001) Atlas of clinical fungi. ASM Press.

Hyvärinen A, Vahteristo M, Meklin T et al. (2001) Temporal and spatial variation of fungal concentrations in indoor air. Aerosol Sci Technol; 35: 688-95. 
Jacobs J, Borràs-Santos A, Krop E et al. (2014) Dampness, bacterial and fungal components in dust in primary schools and respiratory health in schoolchildren across Europe. Occup Environ Med; 71: 704-12.

Kanchongkittiphon W, Mendell MJ, Gaffin JM et al. (2015) Indoor environmental exposures and exacerbation of asthma: an update to the 2000 review by the Institute of Medicine. Environ Health Perspect; 123: 6-20.

Kawel N, Schorer GM, Desbiolles L et al. (2011) Discrimination between invasive pulmonary aspergillosis and pulmonary lymphoma using CT. Eur J Radiol; 77: 417-25.

Krop EJM, Jacobs JH, Sander I et al. (2014) Allergens and $\beta$-glucans in dutch homes and schools: characterizing airborne levels. PLoS One; 9: e88871.

Madsen AM, Matthiesen CB, Frederiksen MW et al. (2012) Sampling, extraction and measurement of bacteria, endotoxin, fungi and inflammatory potential of settling indoor dust. J Environ Monit; 14: 3230-9.

Marcoux D, Jafarian F, Joncas V et al. (2009) Deep cutaneous fungal infections in immunocompromised children. $J \mathrm{Am}$ Acad Dermatol; 61: 857-64.

McGinnis MR. (2004) Pathogenesis of indoor fungal diseases. Med Mycol; 42: 107-17.

Méheust D, Le Cann P, Reboux G et al. (2013) Indoor fungal contamination: Health risks and measurement methods in hospitals, homes and workplaces. Crit Rev Microbiol. 40: 248-60

Mendell MJ, Mirer AG, Cheung K et al. (2011) Respiratory and allergic health effects of dampness, mold, and dampness-related agents: a review of the epidemiologic evidence. Environ Health Perspect; 119: 748-56.

Normand A-C, Vacheyrou M, Sudre B et al. (2009) Assessment of dust sampling methods for the study of cultivable-microorganism exposure in stables. Appl Environ Microbiol; 75: 7617-23.

Normand A-C, Sudre B, Vacheyrou M et al. (2011) Airborne cultivable microflora and microbial transfer in farm buildings and rural dwellings. Occup Environ Med; 68: $849-55$

Noss I, Doekes G, Sander I et al. (2010) Passive airborne dust sampling with the electrostatic dustfall collector: optimization of storage and extraction procedures for endotoxin and glucan measurement. Ann Occup Hyg; 54: 651-8.

Noss I, Wouters IM, Visser M et al. (2008) Evaluation of a low-cost electrostatic dust fall collector for indoor air endotoxin exposure assessment. Appl Environ Microbiol; 74: 5621-7.

Piecková E, Wilkins K. (2004) Airway toxicity of house dust and its fungal composition. Ann Agric Environ Med; 11: 67-73.

Pitkäranta M, Meklin T, Hyvärinen A et al. (2008) Analysis of fungal flora in indoor dust by ribosomal DNA sequence analysis, quantitative PCR, and culture. Appl Environ Microbiol; 74: 233-44.

Portnoy JM, Barnes CS, Kennedy K. (2004) Sampling for indoor fungi. J Allergy Clin Immunol; 113: 189-98; quiz 199.

Reboux G, Bellanger AP, Roussel S et al. (2009) Indoor mold concentration in Eastern France. Indoor Air; 19: 446-53.

Rocchi S, Reboux G, Frossard V et al. (2015a) Microbiological characterization of 3193 French dwellings of Elfe cohort children. Sci Total Environ; 505: 1026-35.

Rocchi S, Richaud-Thiriez B, Barrera C et al. (2015b) Evaluation of mold exposure in cystic fibrosis patients' dwellings and allergic bronchopulmonary risk. J Cyst Fibros. 14: 242-47

Roussel S, Reboux G, Millon L et al. (2012) Microbiological evaluation of ten French archives and link to occupational symptoms. Indoor Air; 22: 514-22.

Samadi S, Heederik DJJ, Krop EJM et al. (2010) Allergen and endotoxin exposure in a companion animal hospital. Occup Environ Med; 67: 486-92.

Tovey ER, Green BJ. (2005) Measuring environmental fungal exposure. Med Mycol; 43: 67-70.

Wålinder R, Ernstgård L, Johanson G et al. (2005) Acute effects of a fungal volatile compound. Environ Health Perspect; 113: $1775-8$.

Zahradnik E, Sander I, Kendzia B et al. (2011) Passive airborne dust sampling to assess mite antigen exposure in farming environments. J Environ Monit; 13: 2638-44.

Zuraimi MS, Fang L, Tan TK et al. (2009) Airborne fungi in low and high allergic prevalence child care centers. Atmos Environ; 43: 2391-400. 